- Tooth transplantation is a rare treatment modality (autotransplantation).

- Calcium hydroxide therapy can assist in bony regeneration following this type of surgical procedure.

- This paper describes a case history involving both surgical and endodontic treatments.

\title{
Calcium hydroxide therapy and bony regeneration following autogenous tooth transplantation: case report and three year follow up
}

\author{
J. Heer ${ }^{1}$
}

\begin{abstract}
Tooth transplantation may be the earliest form of transplantation, possibly carried out by the ancient Egyptians. The surgical technique has changed very little since its documentation in 1954 by M. L. Hale. Autogenous transplantation is the movement of one tooth from one location in the mouth to another in the same individual. ${ }^{1}$ Occasionally it is used to reposition teeth that have developed into an incorrect position. This is occasionally carried out for maxillary canines, but lower molar teeth are also considered, particularly when replacing a lower first permanent molar with a lower third molar. This case report focuses on the autotransplantation ${ }^{1}$ of a maxillary canine taken from the palate and surgically repositioned into the edentulous canine space. By using this technique a prosthetic method such as a dental implant or bridge was avoided.
\end{abstract}

A major advantage of such a technique is that optimal aesthetics are obtained by using the patient's natural tooth and this will have advantages over any form of prosthetic tooth. Fixed prostheses will always impart a detrimental effect on the adjacent teeth from tooth preparation and a removable prosthesis could predispose to long term periodontal problems particularly if maintenance is poor. Dental implant therapy is the other restorative option, but again there is a prosthetic component to restore the edentulous tooth space. ${ }^{2}$ This case study focuses on the postoperative dental management of the patient and not the

${ }^{1}$ General Dental Practitioner \& Oral Surgery Clinical Assistant, JH Dental Clinic, 279 Mitchell Ave, Coventry, CV4 8DU

Correspondence to: Dr Jatinder Heer

Email:jheer@talk21.com

\section{Refereed Paper}

Accepted 20 March 2007

DOI: $10.1038 /$ bdj.2007.894

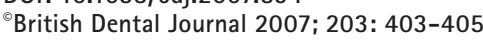

surgical method of transplantation which had been carried out by a consultant maxillofacial surgeon.

\section{CASE REPORT}

This 18-year-old female patient was referred by her general dental practitioner for treatment of a retained mobile deciduous canine and a palatally buried successor (23). After assessment with a consultant oral and maxillofacial surgeon and consultant orthodontist, a decision was made to surgically expose the canine and transplant it from the palate into a prepared tooth socket into the desired position. The tooth was considered unsuitable for surgical exposure alone and orthodontic movement by the joint consultants clinic.

The transplantation procedure was carried out under a general anaesthetic and this involved careful exposure and atraumatic removal. In order to achieve this sufficient bone removal was required to enable complete tooth removal with minimal disturbance to the tooth root and periodontium (although some degree of damage is inevitable). The tooth crown follicle is also completely removed and curettage carried out. Following the transplantation, the tooth was splinted by means of a fixed acrylic splint for two weeks. The splint did allow some slight movement of the tooth following surgery and was constructed by the maxillofacial department. It was a soft acrylic splint. Post-operatively there is little chance of any revascularisation even in immature teeth. However, some form of pulpal vitality testing could be of use where a reliable technique is employed. Such a technique would be the laser Doppler probe, but the cost of such a device is such that it is not practical for use in general practice.

The patient was also kept on a soft diet during the initial week after surgery. The tooth was also reduced palatally at the time of surgery to keep it in infra-occlusion in the initial stages prior to its eruption into occlusion in the months following transplantation. 
After an initial three-month review period, the tooth exhibited some residual mobility. This was as anticipated due to the bony defect that surrounded the root as a result of surgery and postoperative bony resorption.

\section{Calcium hydroxide therapy}

Some form of endodontic treatment was therefore advocated and the patient referred to our general practice for assessment and possible treatment for bony regeneration. There was also some external root resorption noted, affecting the apical third of the tooth root.

\section{Treatment plan}

1. Calcium hydroxide therapy

2. Root canal treatment

3. Consideration to bone augmentation.

From the dental examination, there were no noted occlusal factors exacerbating the Grade I mobility of the tooth. There was no evidence of occlusal trauma or periodontal disease. The periapical radiograph did indicate a radiolucency affecting the tooth root mesially along its entire length (Fig. 1). Vitality testing was not advocated due to unreliability of the testing methods and also due to the fact that root treatment was clearly required from the radiographic findings.

The patient was referred to the general practice at three months post-op. It is, however, ideal to extirpate such teeth at three weeks post-op. It is very unlikely that a tooth with a fully formed apex will revascularise the whole pulp chamber. Andreasen $^{4}$ suggests only the apical 2 $\mathrm{mm}$ is likely, which leaves necrotic pulp in the remaining area of the pulp chamber which could act as a nidus for progression of external inflammatory resorption. Most clinicians would extirpate pulps in fully formed teeth early around the time of splint removal at 2-3 weeks; however this was not possible (due to the timing of the referral). Some resorption in this case was seen on Figure 2 after three months and extirpation was carried out at this time only as the patient was seen on a referral basis and was only referred at three months post-op.

Local anaesthesia was applied and an access cavity cut into the palatal aspect of the canine. A rubber dam was used to isolate the tooth and to prevent ingress of saliva into the root canal as this would lead to the possible ingress of infection and delayed healing. (Some endodontists suggest that once the tooth to be treated has been isolated the operator should change to a new pair of gloves due to the risk of saliva contamination to the canal via the instruments.) Calcium hydroxide is presented in a flowable prepared form commercially available as Hypocal ${ }^{\circledR}$, and once the canal has been cleaned, shaped and dried, this material can be expressed directly into the tooth root and a spiral filler used to act as an archemedian screw and flow the paste into an apical direction. The highly alkaline environment has the following advantages:

a) it is bacteriostatic

b) it inhibits the acid phosphatase enzyme

c) it promotes activity of the alkaline phosphatase enzyme.

There are conflicting reports about the time periods required for this agent to act. For the purposes of this case, the patient had been advised that an approximate time scale of 12-18 months would be required to get some bony regeneration, and the dressing was changed at the following time periods:

1. Every two weeks for ten weeks ${ }^{3}$

2. Every month for three months. ${ }^{3}$

The tooth root was cleaned and irrigated at each dressing change using an endosonic root canal file. The canal was not irrigated at any stage with sodium hypochlorite as the bacteriostatic effect of the calcium hydroxide was sufficient and there was no evidence of pus or any infection during the treatment course.

\section{Endodontic therapy}

This was needed for long term stability of the root and periapical bone health and should be carried out between 36 months post-transplantation. ${ }^{3}$ Canal obturation would prevent the ingress of tissue fluid as the lack of periapical bone would mean that there was a communication to the bony defect via the system of lateral canals. It was this very lateral canal system that we were relying on to transfer the alkaline effect of the calcium hydroxide based dressing and to enable the material to perfuse through and infiltrate the defect.

The canal was filed using endosonic files, K-flex files and a step back method was advocated using an apex

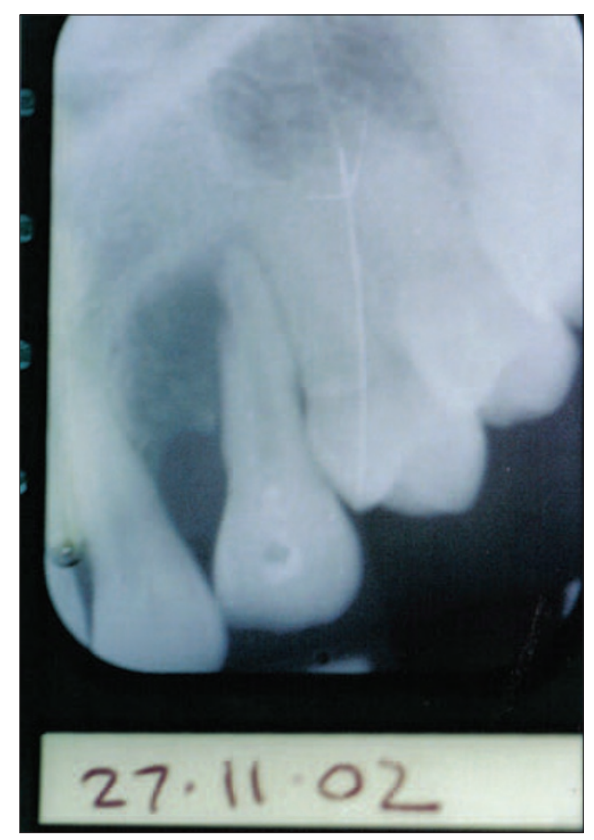

Fig. 1 Periapical radiograph taken after the first three months of calcium hydroxide therapy

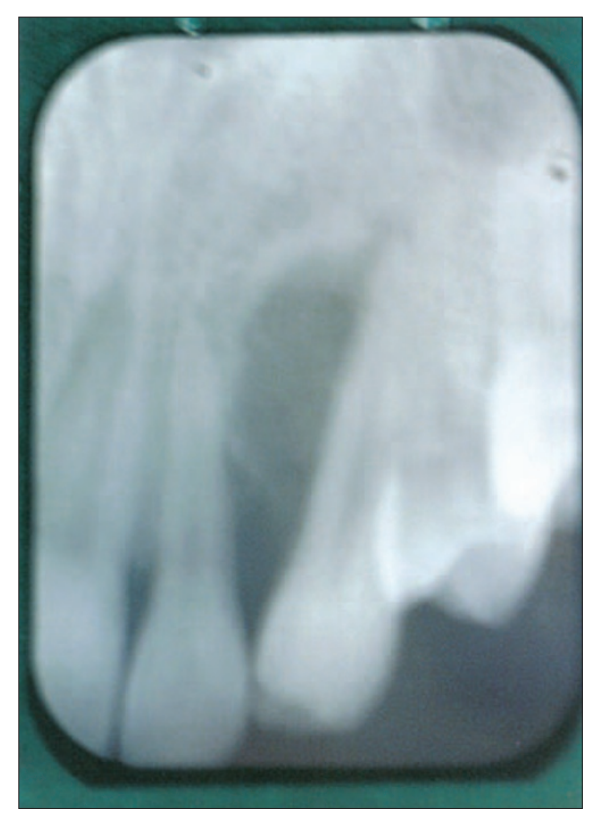

Fig. 2 Initial periapical radiograph taken two weeks post-transplantation

locator. Gates-Glidden burs were used in sequence to carry out coronal flare of the canal. Cold gutta percha ${ }^{3}$ was used and a zinc oxide/eugenol soluble root sealing agent used to seal any residual spaces. Radiography could not be used during pregnancy and was deferred.

\section{Medical history}

Six months into treatment this patient became pregnant and endodontic treatment was carried out without radiographic assistance. The post-operative radiographs were therefore delayed until after the pregnancy. Any subsequent 
changes to the root filling could be implemented at that time if they were required.

\section{RESULTS}

Radiographic evaluation was carried out by means of periapical radiographs taken in a standardised way using the same type of film holder and beam aiming device. The radiographic exposure was also kept constant along with identical film processing (automated Velopex ${ }^{\circledR}$ ), in order to reduce all the possible variables which could affect the radiographic appearance (Figs 3a and 3b). Radiographic evaluation was undertaken at six months and again at three years post-operatively. There was significant bony deposition and the tooth mobility was reduced. There were no periods of infection or discharge.

\section{DISCUSSION}

The radiographic appearance, along with the clinical findings at three years postsurgery, show that autogenous tooth transplantation is a successful treatment modality. Transplantation of a tooth with a mature root and closed apex will usually lead to a loss of vitality and the surgical transplantation technique plays the largest role in long term success. Failures when they occur appear to be related to inflammatory external root resorption. Damage to the periodontal ligament at the time of transplantation appears to play a vital role in this. The tooth is not handled from the root and the transplant is carried out with the tooth extracted in an atraumatic way and by keeping it outside of the mouth and stored in saline for as short a period as possible.

The most important criteria for success are adequate bony support at the recipient site, ${ }^{1}$ and the absence of acute or chronic inflammation. ${ }^{2}$ Immature tooth roots have been documented to produce good results when compared to the results for mature teeth with fully formed apices. The mature teeth will also require root canal therapy whereas their immature counterparts generally do not.

\section{CONCLUSION}

Autotransplantation and calcium hydroxide therapy offer patients an excellent treatment modality where they can achieve excellent aesthetics and function. With the recent publicity surrounding dental implant therapy, transplantation may provide superior aesthetics. Although initial reports suggested disappointing long term results, more recent studies suggest that this treatment modality is at least as successful as dental implant therapy, ${ }^{1}$ based on $85 \%$ at five years and $80 \%$ at ten years. In some cases it may be considered in patients with a history of hypodontia or trauma.

The surgical technique is the most influential factor in long term success and damage to the periodontal ligament will predispose to ankylosis and probable long term inflammatory root resorption. The calcium hydroxide therapy will help to reduce this but long term such resorption processes cannot be effectively prevented.

Auto-transplantation is a rare treatment modality which may be considered in patients who fit the narrow selection criteria. The results achievable are outstanding although there are few long term studies that report success rates over $75 \%$. Since many suitable candidates are teenagers, autotransplantation may be considered in the interim period, prior to dental implant therapy, which may be suitable once the dentition has fully developed in adulthood or if the transplant fails. Autotransplantation is a technique often overlooked due to the surgical difficulty involved and the lack of data supporting long term success.

1. Clokie C, Yau D, Chano L. Autogenous tooth transplantation: an alternative to dental implant placement. J Can Dent Assoc 2001; 67: 92-96.

2. Northway W M, Konigsberg S. Autogenic tooth transplantation: the state of the art. Am J Orthod

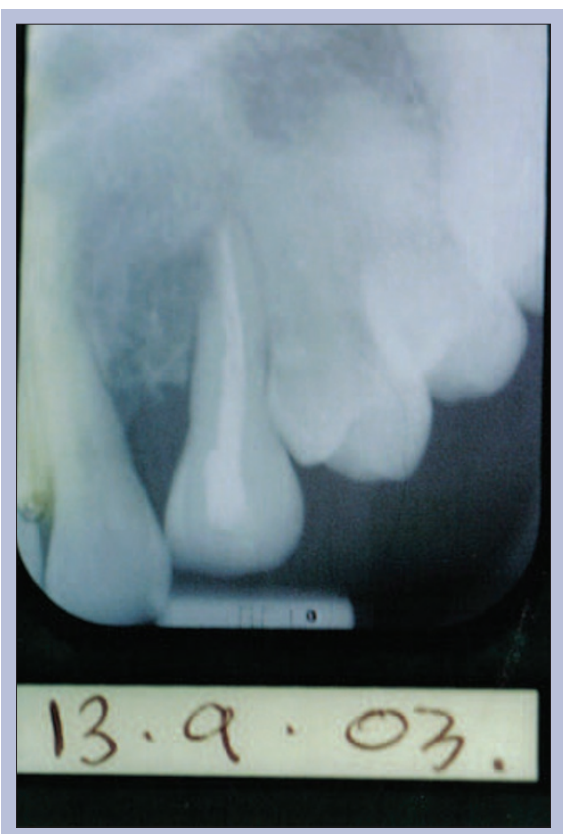

Fig. 3a Master cone radiograph taken at three months

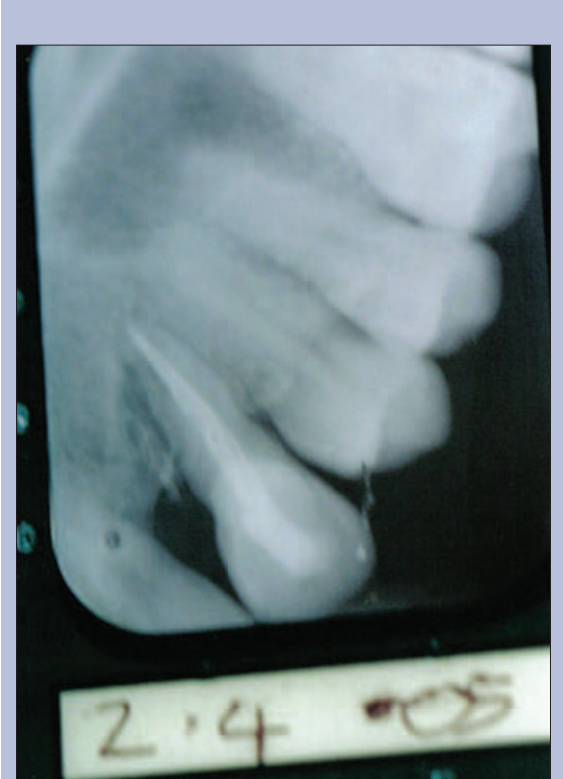

Fig. 3b A postoperative radiograph following root canal therapy at 12 months post-transplantation

1980; 77: 146-162.

3. Nethander G. Periodontal conditions of teeth autogenously transplanted by a two-stage technique. J Periodontal Res 1994; 29: 250-258.

4. Andreasen J 0. Atlas of replantation and transplantation of teeth. Germany: MediGlobe, 1992. 